\title{
Predicción basada en redes neuronales de la resistencia a compresión a los 7 días de los UHPC incorporando SCM
}

\author{
Neural network-based prediction of 7-days \\ compressive strength of UHPC incorporating SCM
}

Joaquín Abellán García ${ }^{1}$

\author{
${ }^{1}$ Escuela Colombiana de Ingeniería Julio Garavito, AK 45 (Autonorte) N.205-59, Bogotá, Cundinamarca, Colombia \\ e-mail: joaquin.abellan-g@escuelaing.edu.co
}

\section{RESUMEN}

El hormigón de ultra alto rendimiento (UHPC) es un tipo de hormigón de alta tecnología que exhibe excelentes propiedades mecánicas y de durabilidad. En los últimos años, el uso de materiales cementantes suplementarios (SCM) como sustitución parcial del humo de cemento y sílice ha sido objeto de gran interés por parte de la comunidad científica para reducir los altos costos y la huella de carbono del UHPC. Algunas sus aplicaciones, como el refuerzo sísmico de estructuras existentes no dúctiles, requieren del desarrollo de resistencias tempranas. Sin embargo, la sustitución del cemento y humo de sílice puede modificar de algunas propiedades, como las resistencias iniciales de UHPC. Por otro lado, el uso de SCM produce un material altamente complejo, siendo más difícil comprender el efecto de cada componente y sus interacciones en el desarrollo de resistencias tempranas en el hormigón. Este estudio tiene como objetivo desarrollar un modelo de redes neuronales artificiales (ANN) para predecir la resistencia a la compresión a los siete días del UHPC, pudiendo incorporar varios SCM como el humo de sílice, ceniza volante, escoria granulada de alto horno, polvo de vidrio reciclado, ceniza de cascarilla de arroz, residuo de catalizador de craqueo catalítico fluido, metacaolín, carbonato cálcico pulverizado, además de filler mineral como el polvo de cuarzo. Para el desarrollo del modelo de una sola capa oculta se usaron 523 datos de investigaciones publicadas. Además, el modelo también fue validado mediante el uso de trabajos experimentales. Finalmente, el algoritmo Connection-WeightApproach (CWA) se utilizó para analizar las relaciones entre los componentes del UHPC y la resistencia a la compresión a los siete días. Los resultados señalaron que el modelo ANN es un modelo eficiente para predecir la resistencia a la compresión a los 7 días del UHPC incluso cuando se incorporan SCM.

Palabras-clave: Validación cruzada k-fold, ANN, UHPC, materiales cementantes suplementarios, resistencia a la compresión a los 7 días.

\begin{abstract}
Ultra-high-performance concrete (UHPC) is a high-tech kind of concrete which exhibits superb mechanical properties and improved durability. Over the last years the use of supplementary cementitious materials (SCM) as partial substitution of cement and silica fume has been the object of great interest by the scientific community in order to reduce the high costs and carbon footprint of UHPC. Some of the more promising applications of this type of special concrete, such as the seismic retrofitting of non-ductile existing structures, require the development of high or ultra-high early strength. However, the replacement of cement and silica fume can result in the modification of some properties such as the early strength of UHPC, which usually needs great amounts of cement and silica fume. On the other hand, the use of several SCM has as outcome a highly complex material, which makes it more difficult to understand the effect of each component and their interactions on early strength. This study is aimed to develop an artificial neural networks (ANN) approach to predict the seven-day compressive strength of UHPC, being able to incorporate several SCM such as silica fume, fly ash, ground granulated blast furnace slag, recycled glass powder, rice husk ash, fluid catalytic cracking catalyst residue, metakaolin, limestone powder, in addition to mineral filler such as quartz powder. A total of 523 data from previous published works was used to train the one-hidden-layer ANN model. The model was also validated by performing experimental works. Besides, Connection-Weight-Approach algorithm (CWA) was used to analyse the relationships between the UHPC's components and the seven-day
\end{abstract}


compressive strength. The results pointed out that the ANN is an efficient model for predicting the early strength (7-day compressive strength) of UHPC even when SCM are incorporated.

Keywords: K-fold validation, ANN, UHPC, supplementary cementitious materials, 7-day compressive strength.

\section{INTRODUCIÓN}

En los últimos años, el desarrollo de los hormigones de alto rendimiento ha hecho un enorme progreso en la industria de la construcción en todo el mundo, lo que ha llevado a nuevos materiales, como el hormigón de ultra alto rendimiento (UHPC, por sus siglas en inglés) [1-5]. El UHPC se define como un hormigón de alta tecnología, con propiedades mecánicas sobresalientes, como una resistencia a la compresión igual o superior a los $150 \mathrm{MPa}$ [6-9], y una durabilidad mejorada debido a su baja porosidad [8, 10, 11]. Una dosificación típica de UHPC incluye cemento Portland (CEM), humo de sílice (SF), polvo de cuarzo (QP), arena silícea (SS) con un tamaño máximo de $600 \mu \mathrm{m}$, superplastificante reductor de agua de alto rango (HRWR), y habitualmente fibras de acero [12-14]. La inclusión de fibra en el UHPC aporta propiedades tan importantes como son la ductilidad y tenacidad del material [15-18].

Sin embargo, en términos de economía y sostenibilidad, el UHPC aún debe ser evaluado con respecto a su coste y huella de carbono en relación con el hormigón convencional [19]. Una dosificación de UHPC generalmente contiene más de $800 \mathrm{~kg} / \mathrm{m}^{3}$ de cemento Portland además de altas cantidades de humo de sílice, cuya finalidad es rellenar los huecos entre las partículas de cemento mejorando la densidad de empaquetamiento del concreto [12-14]. Si bien el alto contenido de cemento aumenta la huella de carbono del UHPC, la necesidad de grandes cantidades de humo de sílice, cuyo costo es más alto que el del cemento, aumenta el precio final del hormigón. Para lograr un material menos costoso y más sostenible, varias investigaciones se han centrado en el uso de materiales cementantes suplementarios como sustitución parcial del cemento y humo de sílice [3, 20-22]. Sin embargo, la reducción en las cantidades de cemento y humo de sílice podría resultar en la modificación de ciertas propiedades, como la resistencia a edades tempranas del UHPC [22]. Los hormigones de resistencia temprana alta y ultra alta a menudo se logran agregando altas cantidades de cemento, humo de sílice o nano sílice, cuyo costo es incluso mayor que el del humo de sílice [23, 24].

En la actualidad, las aplicaciones de UHPC abarcan la construcción de puentes peatonales, plataformas marinas, muros prefabricados, mobiliario urbano, dovelas prefabricadas para excavación con tuneladora, revestimiento en pavimentos dañados y pisos industriales, entre otros [16, 25-30]. Además, entre las aplicaciones más prometedoras de este tipo de material destacan la rehabilitación de estructuras y el refuerzo sismo resistente de estructuras existentes no dúctiles [31-33]. Estas aplicaciones de refuerzo generalmente precisan de hormigones que desarrollen altas o ultra altas resistencias a edades tempranas minimizando riesgos de colapso, ayudando a que la estructura reparada/mejorada entre en servicio lo antes posible, y evitando la demora excesiva en la retirada de los equipos auxiliares de apuntalamiento [31].

El objetivo principal de este trabajo de investigación fue desarrollar un modelo basado en redes neuronales artificiales (ANN) para predecir la resistencia a la compresión a siete dias del UHPC. El modelo ANN fue diseñado de tal manera que permite la consideración de combinaciones de una amplia gama de SCM, incluyendo humo de sílice (SF), cenizas volantes (FA), escoria granulada de alto horno (GBFS), polvo de vidrio reciclado (GP), ceniza de cáscara de arroz (RHA), residuo de craqueo catalítico usado (FCC), metacaolín (MK) y carbonato cálcico pulverizado (LP) además de polvo mineral como el polvo de cuarzo (QP). Esta herramienta matemática podría ser útil em el desarrollo de nuevas dosificaciones de UHPC con reducidas cantidades de materiales caros como el humo de sílice o la nano sílice y con necesidades especiales de resistencia a edades tempranas, incorporando SCM disponibles en el mercado local.

La validación cruzada tipo $k$-fold se utilizó durante la sección del número de neuronas en la capa oculta, así como en la determinación del número de veces (epoch) que se pasan los datos de entrenamiento por la red neuronal. Se utilizó el análisis CWA (Connection-Weight-Approach) [34-36] para, una vez ajustado el modelo, determinar el efecto de cada componente o relación en la resistencia a compresión a los 7 días del UHPC. Además, se realizó una validación experimental para evaluar la precisión del modelo y su habilidad para la predicción en un caso real.

\section{MATERIALES Y METODOLOGÍA}

\subsection{Base de datos}

2.1.1 Recopilación de datos

Las bases de datos de dosificaciones y resistencias a la compresión a los 7 días de los UHPC con diferentes combinaciones de materiales cementantes suplementarios se extrajeron de varios simposios internacionales 
sobre hormigones de alto y ultra alto rendimiento (incluidos los simposios internacionales celebrados en Kassel [37-40]), tesis doctorales, y otros artículos publicados en revistas internacionales. Solo se consideraron para la base de datos aquellas dosificaciones con información de la densidad de empaquetamiento virtual [41, 42] o los datos necesarios para estimarla. El concepto de densidad de empaquetamiento virtual (VPD) se basa en la máxima densidad del hormigón sólo alcanzable si las partículas se colocan una por una. Se trata por lo tanto de un valor teórico y no practico [41-43].

Debido al hecho de que las resistencias a compresión reportadas en los diferentes documentos revisados se basaron en una amplia gama de geometrías y dimensiones de cuerpos de prueba, los valores de la resistencia incorporados a la base de datos se transformaron a equivalente a cubo de $100 \mathrm{~mm}$ de lado por medio de coeficientes de transformación [44-47]. Los coeficientes de transformación utilizados se muestran en la Tabla 1.

Tabla 1: Coeficientes de conversión para las resistencias a compresión, según dimensiones y geometrías de los cuerpos de prueba.

\begin{tabular}{l|l|l|l|l|l|l|l|l|l}
\hline GEOMETRÍA & CUBO & CUBO & CUBO & CUBO & CUBO & CILINDRO & CILINDRO & CILINDRO & CILINDRO \\
\hline DIMENSIONES & 150 & 100 & 70 & 50 & 40 & $50 \times 100$ & $75 \times 150$ & $100 \times 200$ & $150 \times 300$ \\
\hline COEFICIENTE & 1.119 & 1.000 & 0.935 & 0.962 & 1.121 & 1.020 & 1.020 & 1.020 & 1.063 \\
\hline
\end{tabular}

\subsubsection{Detección de datos anómalos}

La detección y tratamiento de valores atípicos es importante para construir un modelo preciso que obtenga resultados adecuados, ya estos datos anómalos pueden influir mucho en el modelo resultante [48]. Por lo tanto, se realizó un análisis de estadística descriptiva de cada variable para identificar posibles valores atípicos [49]. Los errores de entrada de datos, valores atípicos y distribuciones inusuales (datos asimétricos) en la base de datos se identificaron mediante diagramas bagplot. El diagrama bagplot es un diagrama de dispersión que utiliza una generalización bivariada del diagrama Box and whisker plot de Tukey también conocido como "diagrama de caja" o "diagrama de cajas y bigotes". Estos diagramas se emplean para detectar datos inconsistentes y valores atípicos mediante métodos robustos, dibujando elipses que establecen posibles observaciones anómalas [50]. De todos modos, confiar en el uso de esta metodología sin un análisis crítico de los datos también podría ser una práctica peligrosa. Algunos de los datos sospechosos señalados por diagramas bagplot podrían indicar el comportamiento real de los datos, mientras que el resto de las observaciones podrían simplemente agruparse de forma muy próximas. La Figura 1 muestra el diagrama bagplot para el par de variables resistencia a la compresión a los siete días (expresada en MPa) y relación agua cementantes (WB). Es evidente a partir de esta figura que algunas de las dosificaciones que incluyen en su composición de MK (puntos azules) podrían señalarse como valores atípicos (puntos rojos), al ubicarse por fuera de la elipse. Sin embargo, varios estudios han demostrado que la inclusión de MK en el hormigón requiere condiciones especiales de contenido de agua debido a su alto contenido en aluminosilicatos reactivos y la forma amorfa y porosa de sus partículas [51-54]. Por lo tanto, eliminar esas dosificaciones con condiciones especiales en el contenido de agua por hacer fuera de la elipse no sería apropiado.

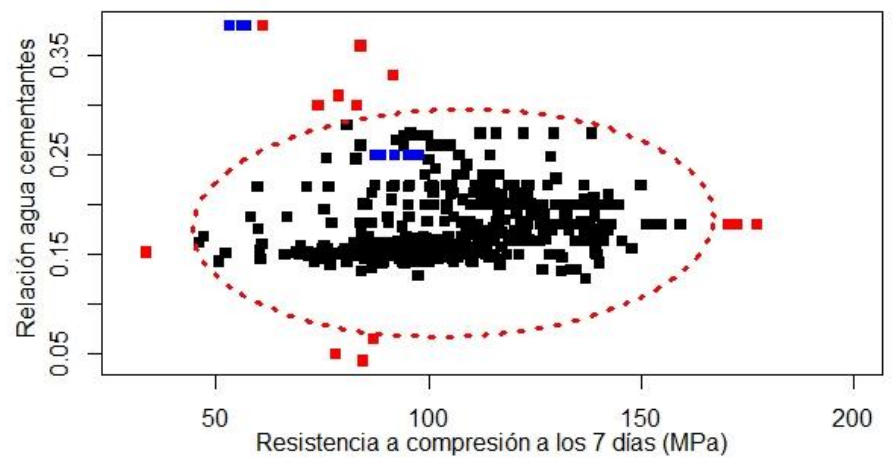

Figura 1: Diagrama bagplot para el par de variables resistencia a la compresión a los siete días (MPa) y relación agua cementantes (WB).

Al final de este proceso, se eliminaron 87 observaciones de la base de datos, dejando 436 para entrenamiento y testeo de los modelos ANN. 


\subsubsection{Normalización de datos}

Antes del desarrollo de los modelos ANN, es necesario realizar la normalización de los datos. Los datos de entrada y salida generalmente comprenden naturalezas y magnitudes diferentes que tienen similitudes nulas o mínimas [55]. Un ejemplo de esto se puede observar en la Tabla 2, donde se aprecia que el contenido de cemento de la base de datos, que está expresado en ratio de volumen total sobre la mezcla, está enmarcado en el rango 0.102-0.567, mientras que el tamaño máximo del agregado, expresado en micrones, está en el rango de 0-19,000. La normalización de los datos elimina la posibilidad de sesgo de la red neuronal hacia las diferentes identidades, escalando todos los datos de entrada y salida. En el presente trabajo de investigación se consideraron dos tipos de normalización. Cuando se empleó la función sigmoidea logarítmica como función de activación, se utilizó una escala lineal en el rango $[0,1]$ que tiene la función que se representa en la ecuación (1). Por otro lado, cuando se empleó la función tangente hiperbólica como función de activación, los datos se escalaron en el rango [-1,1] como se muestra en la ecuación (2):

$$
\begin{aligned}
& x_{\text {norm-sigmoid }}=\frac{x-x_{\min }}{x_{\max }-x_{\min }} \\
& x_{\text {norm-tanh }}=2 \frac{x-x_{\min }}{x_{\max }-x_{\min }}-1
\end{aligned}
$$

donde $x_{\text {norm }}$ es el valor normalizado de la variable $x, x_{\max }$ y $x_{\min }$ son los valores mínimo y máximo de la variable $x$ respectivamente.

2.1.4 División de datos en datos de entrenamiento y datos de testeo

Para evitar problemas de sobreajuste del modelo ANN, los datos recopilados se reordenaron al azar y se dividieron en conjuntos de datos de entrenamiento y testeo. Se utilizaron 326 observaciones con fines de entrenamiento y los 109 restantes se utilizaron para probar los modelos entrenados. Ambos subconjuntos deben contener todos los materiales cementantes suplementarios considerados.

Además, para evaluar la red mientras se ajustan sus hiperparámetros (como el número de neuronas en la capa oculta), los datos de entrenamiento se pueden subdividir en un conjunto de entrenamiento y un conjunto de validación. Sin embargo, dado que hay tan pocas observaciones, el conjunto de validación terminaría siendo muy pequeño para la validación de red neuronal (en nuestro caso dispondríamos de alrededor de 100 datos para esta operación). Por lo tanto, los puntajes de validación pueden cambiar significativamente dependiendo de qué datos fueron seleccionados para la validación y cuáles fueron seleccionados para el entrenamiento. En otras palabras, los puntajes de validación pueden tener una gran variación con respecto a la división de validación. Esto podría llevar a una evaluación poco confiable de la red neuronal [56].

Una solución común para evitar esta situación consiste en aplicar la validación cruzada tipo $k$-fold cuyo esquema se muestra en la Figura 2. Este procedimiento se basa en dividir los datos disponibles en $k$ particiones, crear $k$ modelos idénticos y entrenar a cada uno en $k-1$ particiones mientras evalúa la partición restante. El puntaje de validación para el modelo considerado es entonces el promedio de los $k$ puntajes de validación obtenidos [57-59]. En la presente investigación se consideró validación cruzada tipo $k$-fold con $k=10$.

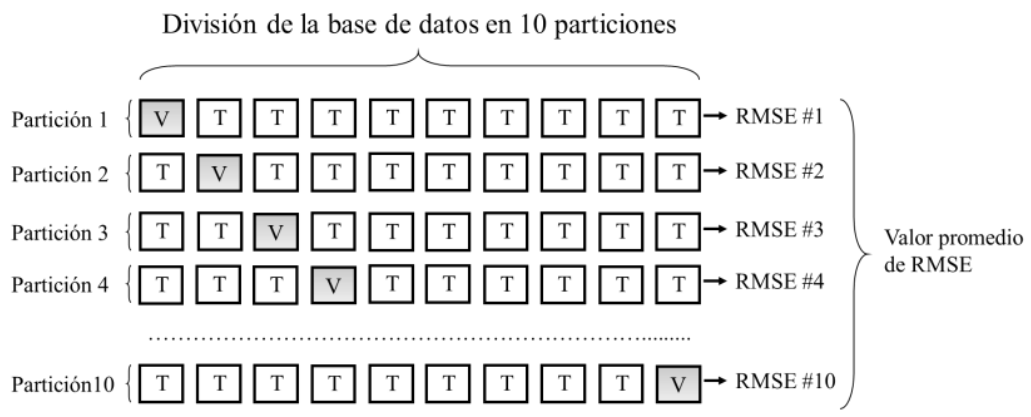

Figura 2: Esquema de la validación cruzada tipo $k$-fold. T: datos de entrenamiento; V: datos de validación. 


\subsection{Investigación analítica}

2.2.1 Redes neuronales artificiales

Los modelos de redes neuronales artificiales se componen de muchas neuronas o nodos de procesamiento altamente interconectados que trabajan conjuntamente [60]. Cada neurona está completamente conectada a la otra a través de pesos de conexión. Las capas sucesivas de neuronas reciben información de las capas anteriores. Las salidas de los nodos en cada capa son entradas a los nodos en la siguiente capa. La forma más simple de arquitectura de red neuronal es el perceptrón. El perceptrón fue desarrollado por Rosenblatt [61] en 1958, y está formado por una neurona con dos entradas y una salida. El diagrama esquemático del perceptrón se

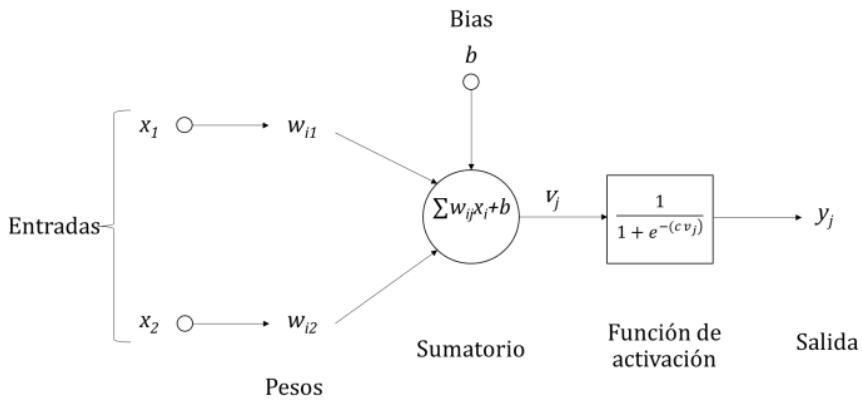

representa en la Figura 3.

Figura 3: Diagrama esquemático del perceptrón.

En la Figura $3 x_{1}$ y $x_{2}$ son las variables de entrada aplicadas a la neurona, y $w_{i 1}$ y $w_{i 2}$ son los pesos de la conexión para cada conexión entrada-neurona oculta. Además, se agrega un término independiente conocido como bias. La función de suma ponderada calcula la entrada neta $\left(v_{j}\right)$ que recibe la neurona [62], tal y como se muestra en la ecuación. (3):

$$
v_{j}=\sum_{i=1}^{n} w_{i j} x_{i}+b
$$

donde $v_{j}$ es la entrada neta de la j-ésima neurona para las señales recibidas de la capa anterior con n neuronas, $w_{i j}$ es el peso entre la $j$-ésima neurona y la $i$-ésima neurona en la capa precedente; $x_{i}$ es la salida de la $i$-ésima neurona en la capa anterior y $b$ es la constante correspondiente a bias [63].

La función de activación procesa la entrada obtenida de la función de suma y determina la salida de la neurona. Algunos ejemplos de funciones de activación incluyen paso, lineal, rampa, tanh (tangente hiperbólica), funciones sigmoideas y relu (función de unidad lineal rectificada) [64]. En este trabajo se evaluó el rendimiento de las funciones tangente hiperbólica y sigmoidea logarítmica como función de activación. La función tangente hiperbólica asigna las entradas entre -1 y +1 [58], mientras que la función sigmoidea logarítmica toma la entrada, que puede tener cualquier valor entre menos y más infinito y comprime la salida en el rango de 0 a 1 [64].

Por otro lado, el ajuste de los pesos de las conexiones de la red neuronal para producir un resultado particular es lo que se denomina "entrenar la red", que es el procedimiento que permite que la red "aprenda" [65]. Rumelhart et al. [65] desarrollaron el algoritmo de aprendizaje más utilizado llamado retropropagación (BP) para redes neuronales del tipo feed-forward. El algoritmo de entrenamiento de BP está basado un gradiente descendiente iterativo diseñado para minimizar el error cuadrado medio entre la salida real de la red neuronal de alimentación de múltiples capas y la salida deseada, que es lo que se conoce como función de coste [66]. En los últimos años se han desarrollado otros algoritmos de aprendizaje. Uno de ellos es el de retropropagación resiliente (Rprop), que tiene dos ventajas principales sobre el algoritmo de BP: Primero, el entrenamiento con Rprop suele converger más rápidamente que el entrenamiento con BP. En segundo lugar, Rprop elimina la influencia dañina del tamaño de las derivadas de error parcial en las iteraciones de ajuste de los pesos, a diferencia del algoritmo de BP que necesita valores para la tasa de aprendizaje. Se recomienda a los lectores interesados que consulten las referencias para la descripción detallada de este algoritmo de aprendizaje $[66,67]$. 
En este estudio, los modelos ANN fueron entrenados usando la retropropagación resiliente como algoritmo de aprendizaje.

\subsubsection{Variables de entrada del modelo ANN}

Se utilizaron diecisiete variables de entrada, incluyendo el contenido en volumen de cemento (C), humo de sílice (SF), ceniza volante (FA), escoria granulada molida de alto horno (GBSF), polvo de vidrio reciclado (GP), cenizas de cascarilla de arroz (RHA) ), residuo catalítico fluido (FCC), metacaolín (MK), carbonato cálcico pulverizado (LP), agua (W), superplastificante reductor de agua de alto rango (HRWR), polvo de cuarzo (QP) y agregado total (A), así como otras propiedades del UHPC, como el tamaño máximo del agregado expresado en micrones (MSA), la relación agua cementantes (WB), la relación agua polvos totales (WP) y la densidad de empaquetamiento virtual (VPD) calculada según De Larrard [14, 41, 68].

El rango de variación de todas las variables contenidas en la base de datos se muestra en la Tabla 2

Tabla 2: Rango de variación y estadísticas de las variables consideradas en este estudio.

\begin{tabular}{l|l|l|l|l|l|l|l|l|l}
\hline & C & SF & FA & GBFS & GP & FCC & MK & LP & W \\
\hline Máximo & 0.567 & 0.155 & 0.250 & 0.247 & 0.202 & 0.177 & 0.091 & 0.161 & 0.190 \\
\hline Mínimo & 0.102 & 0.000 & 0.000 & 0.000 & 0.000 & 0.000 & 0.000 & 0.000 & 0.000 \\
\hline Promedio & 0.246 & 0.063 & 0.016 & 0.008 & 0.027 & 0.004 & 0.005 & 0.002 & 0.025 \\
\hline Desviación Estándar & 0.072 & 0.035 & 0.043 & 0.025 & 0.052 & 0.018 & 0.014 & 0.013 & 0.043 \\
\hline
\end{tabular}

\begin{tabular}{l|l|l|l|l|l|l|l|l}
\hline & HRWR & QP & MSA & WB & WP & A & VPD & $\boldsymbol{f}_{7 d}(\mathrm{MPa})$ \\
\hline Máximo & 0.063 & 0.235 & 19,000 & 0.322 & 0.322 & 0.694 & 0.877 & 159.168 \\
\hline Mínimo & 0.009 & 0.000 & 0.000 & 0.124 & 0.107 & 0.000 & 0.581 & 50.678 \\
\hline Promedio & 0.026 & 0.026 & 1,724 & 0.187 & 0.177 & 0.364 & 0.771 & 105.965 \\
\hline Desviación Estándar & 0.012 & 0.051 & 2,498 & 0.037 & 0.034 & 0.130 & 0.044 & 19.521 \\
\hline
\end{tabular}

\subsubsection{Selección de la arquitectura del modelo ANN}

En esta investigación, se desarrolló un modelo ANN de una capa oculta usando la versión R 3.5.2 (2018-1220) [69] por medio de la función neuralnet [70]. Para cada función de activación, se llevaron a cabo 30 arquitecturas diferentes variando el número de neuronas en la capa oculta desde 1 hasta 30 . Además, para cada una de esas arquitecturas se calcularon 100 modelos diferentes variando la asignación de pesos iniciales del modelo. Los criterios para elegir la asignación de pesos iniciales y el número de neuronas en la capa oculta se centraron en minimizar el error cuadrático medio (RMSE) entre los valores reales y los pronosticados durante la validación cruzada tipo $k$-fold [56] durante el entrenamiento. De este modo, se desarrollaron un total de 3,000 modelos de redes artificiales para predecir la resistencia a la compresión a los 7 días del UHPC.

\subsubsection{Evaluación de desempeño del modelo}

La precisión del modelo ANN desarrollado en este estudio se evaluó utilizando cinco evaluadores de rendimiento estadístico diferentes: la raíz del error cuadrático medio (RSME), la relación entre el RSME y la desviación estándar de los datos medidos (RSR), el error de sesgo medio normalizado (NMBE), la eficiencia de Nash-Sutcliff (E), y coeficiente de determinación múltiple $\left(\mathrm{R}^{2}\right)$ Estos parámetros estadísticos se pueden calcular utilizando las ecuaciones (4) - (8):

$$
\begin{aligned}
& R M S E=\sqrt{\frac{\sum_{i=1}^{n}\left(a_{i}-\hat{a}_{i}\right)^{2}}{n}} \\
& R S R=\sqrt{\frac{R M S E}{\frac{1}{n} \sum_{i=1}^{n}\left(a_{i}-\bar{a}_{i}\right)^{2}}}
\end{aligned}
$$




$$
\begin{aligned}
& \operatorname{NMBE}(\%)=\frac{\frac{1}{n} \sum_{i=1}^{n}\left(a_{i}-\hat{a}_{i}\right)}{\bar{a}_{i}} \times 100 \\
& E=1-\frac{\sum_{i=1}^{n}\left(a_{i}-\hat{a}_{i}\right)^{2}}{\sum_{i=1}^{n}\left(a_{i}-\bar{a}_{i}\right)^{2}} \\
& R^{2}=1-\frac{\sum_{i=1}^{n}\left(a_{i}-\hat{a}_{i}\right)^{2}}{\sum_{i=1}^{n}\left(\hat{a}_{i}\right)^{2}}
\end{aligned}
$$

donde: $a$ es el valor real de la resistencia a la compresión a 7 días en $\mathrm{MPa} ; \bar{a}$ representa el valor promedio de las resistencias reales, $\hat{a}$ es el valor predicho de la resistencia a la compresión a 7 días y $n$ es el número total de observaciones de la base de datos.

El estadístico RMSE es una medida de uso frecuente de las diferencias entre pares de valores [55]. El RMSE compara los valores reales con los estimados y calcula la raíz cuadrada del error residual promedio, lo que indica un error expresado en las unidades de las variables que se comparan, facilitando el análisis de los resultados. Los valores de RMSE de cero indican un ajuste perfecto del modelo. Sin embargo, el RMSE da más peso a los errores grandes [55]; El parámetro estadístico RSR fue presentado por Moriasi et al. [71] e incluye los beneficios de las estadísticas del estadístico RMSE incorporando un factor de normalización (divide por la desviación estándar). A menor valor de RSR mejor es la predicción del modelo; El coeficiente de eficiencia (E), propuesto por Nash y Sutcliffe [72] es uno de los índices estadísticos más ampliamente empleados para evaluar el rendimiento de modelos predictivos. Un valor de E cercano a 1.0 indica una buena precisión del modelo; el NMBE proporciona información sobre el sesgo medio en las predicciones de un modelo. Un NMBE positivo indica una sub-predicción o una predicción media inferior a los valores reales y un NMBE negativo indica una sobre-predicción del modelo [74]. El coeficiente de determinación $\left(\mathrm{R}^{2}\right)$ refleja la bondad del ajuste del modelo a la variable que quiere explicar [74]. Las estadísticas de $\mathrm{R}^{2}$ dependen de las relaciones lineales entre los valores observados y los calculados por el modelo y a veces pueden dar resultados sesgados cuando esta relación no es lineal o cuando los valores contienen muchos valores atípicos. Un valor $\mathrm{R}^{2}$ cercano a la unidad indica una buena asociación entre los valores observados y los calculados. Un uso combinado de los índices de estadísticos expuestos anteriormente puede proporcionar una estimación imparcial de la capacidad de predicción del modelo ANN.

\subsection{Investigación experimental}

Con la finalidad de realizar una validación del modelo predictivo sobre datos experimentales, se realizaron 89 amasadas de hormigones tipo UHPC en laboratorio con diferentes combinaciones de cemento, agua, superplastificante, arena silícea y los materiales cementantes suplementarios expuestos en el punto 2.2.2.

Para mayor información sobre los materiales utilizados, combinaciones consideradas, técnicas, normas y procedimientos de ensayo, se recomienda consultar otras publicaciones del autor $[3,4,20,21,75,76]$.

\section{RESULTADOS Y DISCUSIÓN}

\subsection{Arquitectura de la red neuronal}

Seleccionar la arquitectura del modelo de redes neuronales, en nuestro caso el número de neuronas en la capa oculta, es el primer paso importante en el desarrollo del modelo. La Figura 4 representa el efecto del número de neuronas de la capa oculta en el valor del RMSE obtenido para cada función de activación durante el entrenamiento con validación cruzada tipo $k$-fold. Debe destacarse que la Figura 4 solo representa el valor de RMSE correspondiente a la asignación de pesos iniciales de mejor rendimiento (de entre las 100 probadas) para cada una de las arquitecturas calculadas. De acuerdo con esto, el mejor rendimiento de RMSE se logró cuando se utiliza la función sigmoidea logarítmica como función de activación y se cuenta con tres neuronas en la capa oculta. Por lo tanto, se seleccionó la arquitectura que se muestra en la figura 5. 


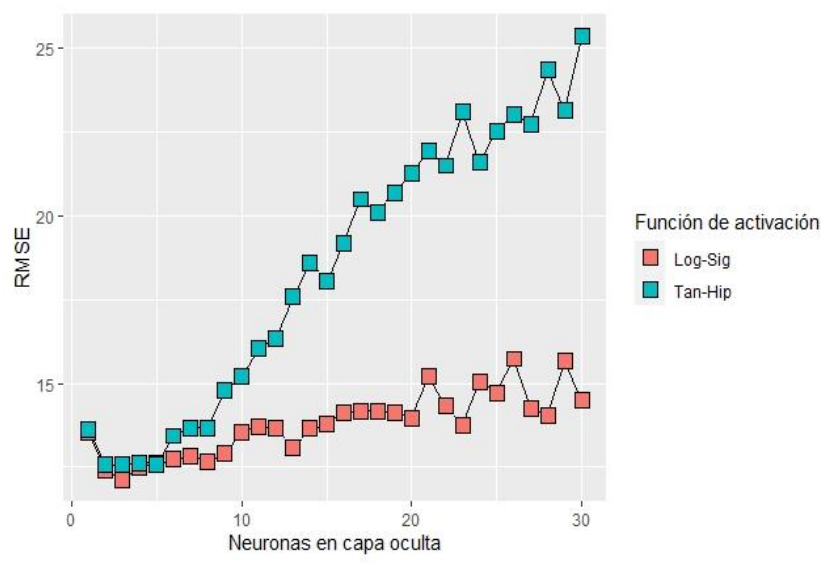

Figura 4: Valor de RMSE versus número de neuronas en capa oculta y función de activación.

Sin embargo, el valor RMSE mínimo de 12.13 MPa alcanzado para esta configuración parece un error todavía muy alto, considerando que la media de los valores de la base de datos es 105.96 MPa (ver Tabla 2). Por lo tanto, es necesario mejorar el rendimiento del modelo. Una forma habitual de mejorar el rendimiento de la red neuronal consiste en pasar los datos de entrenamiento a través de la red varias veces [56]. Para determinar las veces que es necesario pasar los datos de entrenamiento por la red (epochs), se vuelve a utilizar la validación cruzada tipo $k$-fold. La Figura 6 representa una curva suavizada del valor promedio de RSME en la validación $k$-fold versus el número de epochs.

Figura 5: Modelo ANN.

Figura 6: Modelo ANN.
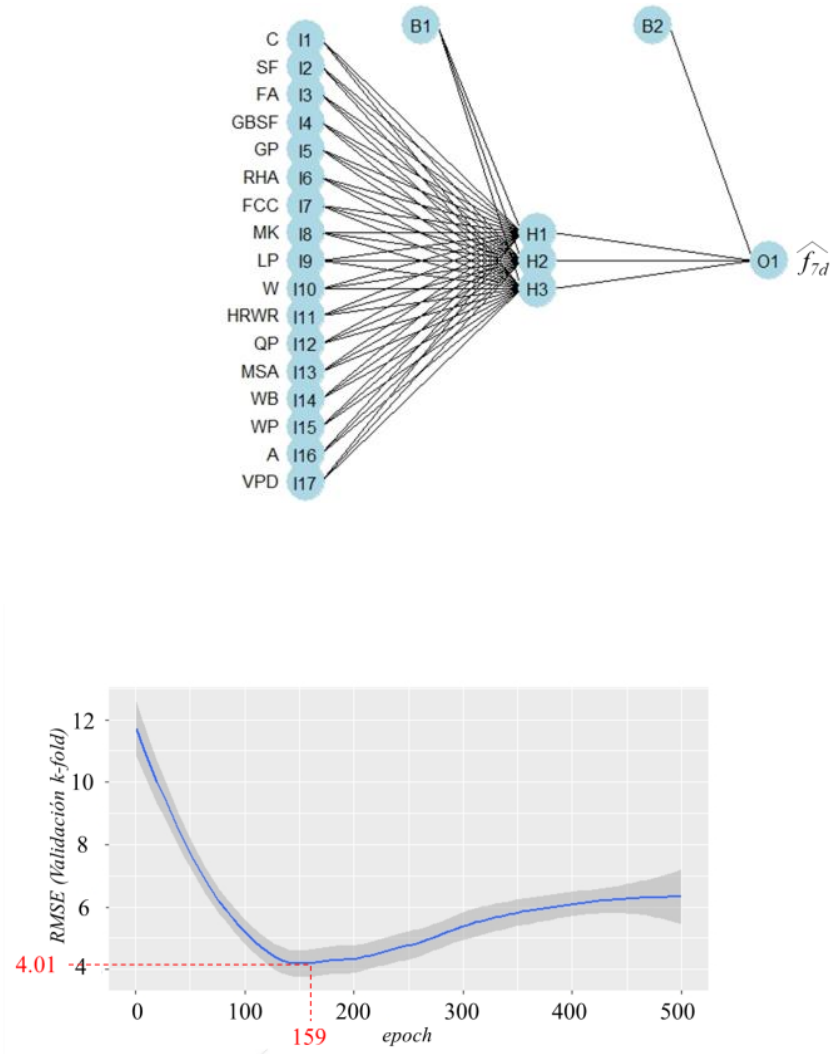

Como puede observarse en la Figura 6, la validación RSME dejó de mejorar significativamente después de 159 epochs para el modelo propuesto. Pasado este punto el modelo comenzó a tener un peor desempeño. Después de este proceso, se puede observar una mejora significativa, habiendo reducido el RMSE obtenido de 12.13 a 4.01 . 


\subsection{Evaluación del modelo ANN}

Los resultados de la evaluación del modelo mediante los índices estadísticos expuestos en el punto 2.2.4 para los datos de entrenamiento, testeo y experimentales se presentan en la Tabla 3.

Tabla 3: Índices estadísticos de evaluación del modelo sobre los datos de entrenamiento, testeo y experimentales.

\begin{tabular}{l|l|l|l|l|l}
\hline DATOS & RMSE & RSR & NMBE (\%) & $\mathbf{E}$ & $\mathbf{R}^{\mathbf{2}}$ \\
\hline Entrenamiento & 4.011 & 0.206 & 0.297 & 0.957 & 0.962 \\
\hline Testeo & 4.447 & 0.277 & $-0.283 \%$ & 0.948 & 0.955 \\
\hline Experimentales & 6.989 & 0.354 & $-1.926 \%$ & 0.874 & 0.898 \\
\hline
\end{tabular}

De acuerdo con la Tabla 3, los indicadores estadísticos usados para la evaluación del modelo indican una precisión similar en los datos de entrenamiento y testeo. Esto confirma el buen desempeño de la validación cruzada $k$-fold empleada como procedimiento para evitar el sobreajuste del modelo ANN [56] durante el proceso de entrenamiento. Por otro lado, hay una depreciación insignificante de los índices cuando se trata de los datos experimentales. El más destacado es el correspondiente al NMBE, que comentaremos más adelante.

El modelo propuesto pronosticó la resistencia a la compresión a 7 días del UHPC en el conjunto de datos de prueba con un valor del estadístico RMSE de 4.447 y $6.989 \mathrm{MPa}$ para los conjuntos de datos de testeo y experimentales, señalando que las diferencias entre los valores predichos y los valores reales de resistencia a la compresión eran relativamente pequeñas. Las estadísticas RSR de 0.277 y 0.354 y los valores E de 0.948 y 0.874 también indicaron un buen desempeño del modelo en los conjuntos de datos de testeo y experimentales. El estadístico NMBE para datos de testeo y experimentales se evaluó como $-0.283 \%$ y $-1.926 \%$. El valor de NMBE medido en el conjunto de datos experimentales señaló una sobreestimación del modelo para los datos experimentales. Esto último también se puede observar en la Figura 7. Este hecho se podría atribuir a la introducción de sesgo debido al uso de un determinado tipo de cemento, agua y superplastificante en los experimentos. Por ejemplo, en la campaña experimental se usó cemento tipo CEM III, mientras que casi todas las dosificaciones de UHPC de la base de datos usaron CEM I y CEM II, que son los recomendados para este tipo de hormigones especiales [77].

Además, se puede observar que el valor de $\mathrm{R}^{2}$ obtenido en los datos de testeo fue similar a los obtenidos por otros autores consultados al pronosticar las propiedades de UHPC usando ANN [75, 78, 79]. Se logró un valor de $\mathrm{R}^{2}$ de 0.955 en el conjunto de testeo usando el modelo propuesto, y, mucho más importante, se logró un valor de $\mathrm{R}^{2}$ de 0.898 en los datos experimentales, lo que indica no solo la buena precisión sino también la versatilidad del modelo al ser utilizado con nuevos materiales. Además, la Figura 7 representa claramente que los valores predichos y los valores reales se encuentran muy próximos en el gráfico en todos los subconjuntos mencionados anteriormente.
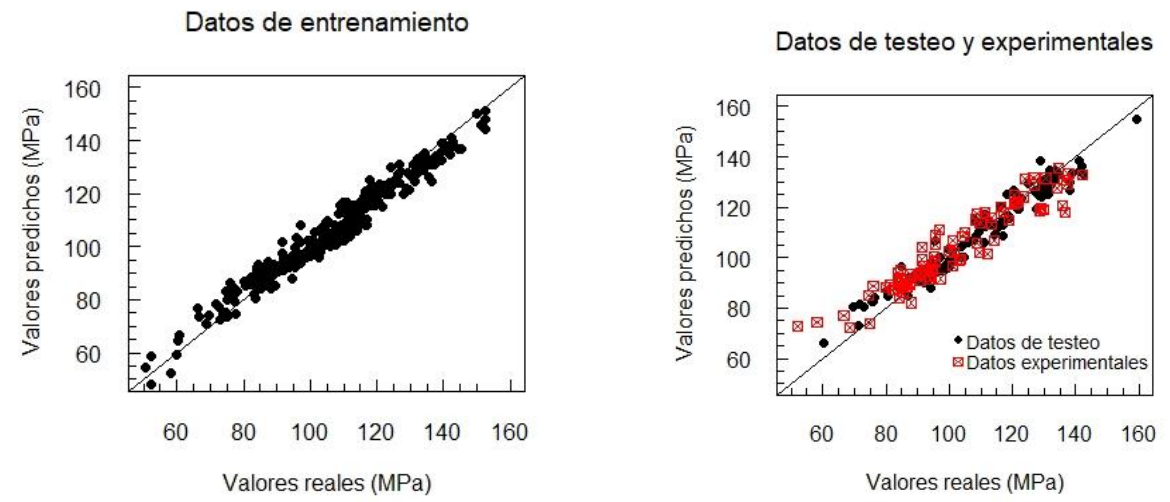

Figura 7: Gráficos de valores reales versus predichos por el modelo para todos los subconjuntos de datos considerados. 


\subsection{Análisis CWA}

Los modelos de redes neuronales generalmente se han etiquetado como black-box, ya que se cree que proporcionan poca información explicativa sobre las contribuciones de las variables independientes en el proceso de predicción. Sin embargo, para explorar la conexión entre las señales de entrada y la salida, Olden et al. [35] desarrollaron el algoritmo CWA. El método CWA utiliza los pesos de las conexiones de la red neuronal, proporcionando una metodología adecuada para cuantificar con precisión la importancia cada variable de entrada en la respuesta final del modelo [34, 35]. Una ventaja de esta metodología es que las contribuciones relativas de cada peso de conexión se mantienen tanto en magnitud como en signo. Por ejemplo, los pesos de conexión que cambian el signo (es decir, positivo a negativo) entre las conexiones de entrada capa oculta y de capa oculta a salida tendrían un efecto de compensación entre ellos. La metodología CWA se utilizó en este estudio para evaluar la importancia de cada variable de entrada en la resistencia a la compresión a los 7 días de los UHPC. Estos análisis son útiles para comprender la influencia de cada uno de los componentes del UHPC en la resistencia a la compresión a los 7 días. Esto último permite poder seleccionar a priori los componentes que mejor se adapten a las propiedades requeridas para las aplicaciones deseadas, reduciendo el número de ensayos innecesarios en la campaña experimental.

Los resultados del análisis CWA se presentan en la Figura 8. Es evidente a partir de esta figura que las variables de entrada con efecto positivo más importantes para la resistencia a 7 días fueron el contenido MK y FCC. Una posible explicación de esto último se puede encontrar en el trabajo de Khatib y Wild [80]. Ellos descubrieron que hay una disminución en la porosidad y el contenido de $\mathrm{Ca}(\mathrm{OH})_{2}$ de las pastas y morteros adicionados con MK en las primeras etapas de hidratación. Además, según varias investigaciones experimentales, debido al elevado contenido en aluminatos reactivos, la adición de MK y/o FCC provoca el desarrollo, en las etapas iniciales de la hidratación, de $\mathrm{C}_{2} \mathrm{ASH}_{8}$ (gehlenita), una pequeña cantidad de fase cristalina $\mathrm{C}_{4} \mathrm{AH}_{13}$, además de favorecer la formación de etringita (especialmente en el caso del FCC), lo que mejora el desarrollo de resistencias tempranas de las pastas y hormigones [20, 75, 81, 82]. Las siguientes variables en importancia positiva serían el contenido de cemento y la densidad de empaquetamiento virtual. La importancia del cemento es obvia, y el caso de VPD concuerda con aquellos estudios que justifican las excelentes propiedades mecánicas del UHPC debido a su estructura ultradensa [6-8]. A continuación, encontramos el SF, siendo que varios estudios han demostrado los beneficios para el desarrollo temprano de la resistencia a través de la adición de humo de sílice debido a la mejora de VPD y su alto contenido en sílice amorfa [83-86]. Por otro lado, el efecto positivo en la resistencia a la compresión a 7 días de LP es consistente con el de estudios previos que encontraron que, especialmente en las primeras edades, el LP mejora la tasa de hidratación del cemento al llenar los poros entre las partículas de cemento debido a la formación de carboaluminato [87, 88]. Además, Ghafoori et al. [88] demostraron que el LP también puede reaccionar con $\mathrm{C}_{3} \mathrm{~A}$ para formar hidratos de monocarboaluminato, lo que aumenta ligeramente la resistencia a la compresión a edades tempranas. Varias investigaciones previas también informaron el efecto positivo de QP sobre la resistencia temprana de UHPC, aunque este efecto aumenta cuando se usan condiciones de curado por calor [90, 91]. Para cerrar las variables con efectos positivos, el análisis CWA muestra que el contenido de GBSF, el volumen total de agregado y el tamaño máximo de agregado tienen un efecto positivo sobre la resistencia, aunque con una importancia más limitada.

Por otro lado, el efecto negativo más significativo en la resistencia a la compresión a los 7 días del UHPC corresponde por orden al contenido de agua, el contenido de superplastificante y las relaciones de agua cementantes y agua polvos totales. El efecto negativo del aumento de agua en la resistencia a la compresión es un efecto bien conocido. Incluso el efecto negativo del superplastificante (HRWR) en el desarrollo temprano de la resistencia ha sido demostrado por varios investigadores [3, 92, 93]. El superplastificante, producido a base de éter a base de policarboxilato, ralentiza la hidratación de los silicatos (especialmente la fase alita) y afecta la formación de ettringita, retardando o perjudicando el desarrollo de resistencias a edades tempranas $[3,21,92]$. El efecto nocivo del contenido de FA en la resistencia temprana del hormigón ha sido reportado por varias investigaciones [77, 81, 94, 95]. Además, el análisis de CWA sugirió que GP parece tener un efecto negativo reducido en la resistencia a la compresión de UHPC. Podría explicarse por su alto contenido de partículas en forma de aguja que podría perjudicar la resistencia a la compresión del hormigón [3]. Finalmente, la inclusión de RHA no aumenta la resistencia a la compresión del hormigón a edades tempranas [96]. Estudios previos han demostrado que el grado de hidratación del cemento mezclado con RHA es bajo en el período inicial [84]. 


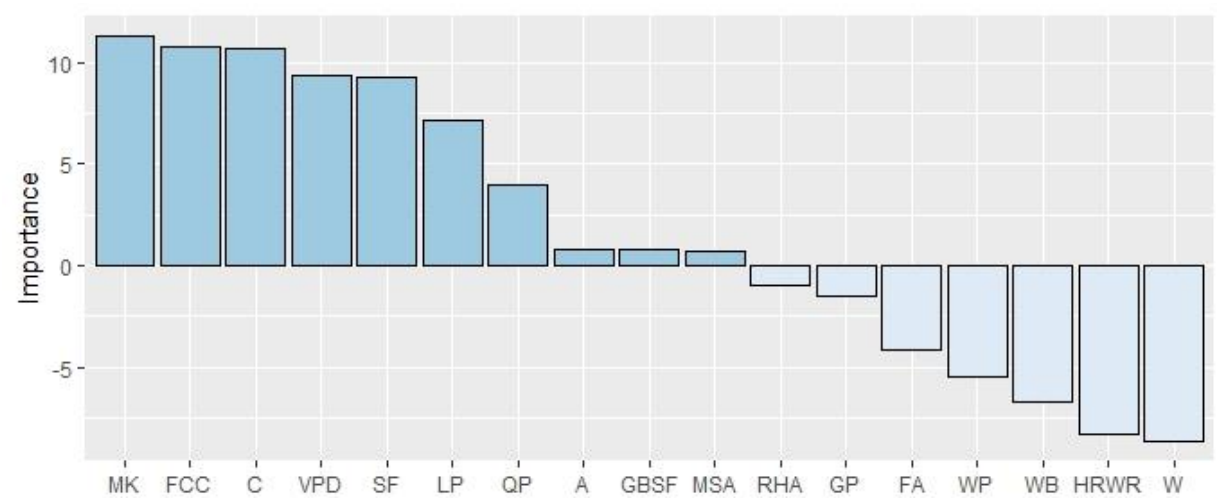

Figura 8: Importancia de las variables de entrada en la resistencia a la compresión de los UHPC a la edad de 7 días.

\section{CONCLUSIONES}

Esta investigación propone un modelo preciso y versátil basado en redes neuronales de una capa oculta para predecir la resistencia a la compresión a los 7 días del UHPC que incorpora SCM. Para evitar el sobreajuste del modelo a los datos de entrenamiento, se utilizó la validación cruzada tipo $k$-fold. En base a los resultados de esta investigación, se extraen las siguientes conclusiones:

1. El modelo propuesto podría ser útil en el desarrollo de UHPC con necesidades de resistencia temprana al reducir los costos y el tiempo en la campaña experimental al permitir preseleccionar las combinaciones de SCM disponibles que tienen mejores resultados en el modelo a menor costo.

2. Los resultados del uso combinado de las métricas de rendimiento, que incluyeron RMSE, RSR, E, NMBE y $\mathrm{R}^{2}$ proporcionaron una información imparcial que confirmó la idoneidad del modelo propuesto.

3. Los resultados de validación obtenidos en la base de datos experimentales $\left(\mathrm{R}^{2}=0.898\right)$ demostraron la versatilidad del modelo para trabajar con una nueva base de datos.

4. Los resultados de la CWA señalaron a MK, FCC y LP como materiales cementantes suplementarios que mejoraron la resistencia inicial de UHPC, además de otros factores como VPD, contenido en cemento y contenido de SF.

5. FA, GP y RHA fueron indicados por la CWA como materiales nocivos para la resistencia a la compresión a los 7 días de los UHPC.

6. El resultado del análisis de CWA mostró coherencia con los resultados de varias investigaciones internacionales. Esto reafirmó la validez del modelo desde un punto de vista científico.

\section{AGRADECIMENTOS}

El autor desea expresar su agradecimiento a los centros de cómputo APOLO de la universidad EAFIT (Medellín-Colombia) y OSIRIS de la Escuela Colombiana de Ingeniería Julio Garavito (Bogotá-Colombia) por el acceso a servidores y el soporte relacionado con los trabajos de computación de los algoritmos. Asimismo, a Cementos Argos SA. por donar la mayoría de los materiales utilizados en la investigación experimental descrita en este documento. Agradecimiento especial para las empresas Cristalería Peldar SA, Ecopetrol SA y Gerdau SA por el suministro de vidrio reciclado, FCC y GBSF para esta investigación.

\section{BIBLIOGRAFIA}

[1] FERDOSIAN, I., CAMÕES, A., "Effective low-energy mixing procedure to develop high-fluidity cementitious pastes," Revista Materia, ISSN 1517-7076 artigo 11676, pp.11-17, 2016

[2] SOLIMAN, N.A., TAGNIT-HAMOU, A., "Partial substitution of silica fume with fine glass powder in UHPC: Filling the micro gap," doi: http://doi.org/10.1016/j.conbuildmat.2017.02.084. Constr. Build. Mater., v. 139 , pp. 374-383, 2017.

[3] ABELLÁN, J., FERNÁNDEZ, J., TORRES, N., et al., "Statistical optimization of ultra-highperformance glass concrete," doi: http://doi.org/10.14359/51720292. ACI Mater. J., v. 117, n. 1, pp. 243-254, 2020 . 
[4] ABELLÁN, J., FERNÁNDEZ, J., TORRES, N., et al. "Development of cost-efficient UHPC with local materials in Colombia," in Proceedings of Hipermat 2020 - 5th International Symposium on UHPC and Nanotechnology for Construction Materials, 2020, pp. 97-98.

[5] SHI, C., WU, Z., XIAO, J., et al. "A review on ultra high performance concrete: Part I. Raw materials and mixture design," doi: http://doi.org/10.1016/j.conbuildmat.2015.10.088. Constr. Build. Mater., v. 101, no. October, pp. 741-751, 2015.

[6] GHAFARI, E., COSTA, H., NUNO, E., et al. "RSM-based model to predict the performance of selfcompacting UHPC reinforced with hybrid steel micro-fibers," doi:

http://doi.org/10.1016/j.conbuildmat.2014.05.064 0950-0618. Constr. Build. Mater., v. 66, n. September, pp. 375-383, 2014,

[7] SCHMIDT, C., SCHMIDT, M., "Whitetopping of Asphalt and Concrete Pavements with thin layers of Ultra-High-Performance Concrete - Construction and economic efficiency," in Proceedings of Hipermat 2012 - 3rd International Symposium on UHPC and Nanotechnology for Construction Materials, 2012.

[8] ABBAS, S., NEHDI, M. L., SALEEM, M.A., "Ultra-High Performance Concrete: Mechanical Performance, Durability, Sustainability and Implementation Challenges," Int. J. doi: http://doi.org/10.1007/s40069016-0157-4. Concr. Struct. Mater., v. 10, n. 3, pp. 271-295, 2016.

[9] ACI COMMITTE 239, "ACI - 239 Committee in Ultra-High Performance Concrete." ACI, Toronto, 2018.

[10] SOLIMAN, N.A., TAGNIT-HAMOU, A., "Using particle packing and statistical approach to optimize eco-efficient ultra-high-performance concrete," doi: http://doi.org/10.14359/51701001. ACI Mater. J., v. 114, n. 6, pp. 847-858, 2017.

[11] JAMMES, F.X., CESPEDES, X., RESPLENDINO, J., "Design of Offshore Wind Turbines", in RILEMfib-AFGC Int. Symposium on Ultra-High Performance Fibre-Reinforced Concrete UHPFRC 2013, 2013, n. 1, pp. 443-452.

[12] TAGNIT-HAMOU, A., SOLIMAN, N.A., OMRAN, A., "Green Ultra - High - Performance Glass Concrete," First Int. Interact. Symp. UHPC - 2016, v. 3, n. 1, pp. 1-10, 2016.

[13] RICHARD P., CHEYREZY, M., "Composition of reactive powder concretes," doi: https://doi.org/10.1016/0008-8846(95)00144-2. Cem. Concr. Res., v. 25, n. 7, pp. 1501-1511, 1995.

[14] DE LARRARD, F., SEDRAN, T., "Mixture-proportioning of high-performance concrete," doi: http://doi.org/10.1016/S0008-8846(02)00861-X. Cem. Concr. Res., v. 32, n. 11, pp. 1699-1704, 2002.

[15] KOU, S.C., XING F., "The Effect of Recycled Glass Powder and Reject Fly Ash on the Mechanical Properties of Fibre-Reinforced Ultrahigh Performance Concrete," doi: http://doi.org/10.1155/2012/263243. Hindawi Publ. Corp. Adv. Mater. Sci. Eng., n. May, 2012.

[16] SOLIMAN, N.A., TAGNIT-HAMOU, A., "Using glass sand as an alternative for quartz sand in UHPC," doi: http://doi.org/10.1016/j.conbuildmat.2017.03.187. Constr. Build. Mater., v. 145, pp. 243-252, 2017.

[17] ABELLÁN-GARCÍA, J., FERNANDEZ-GOMEZ, J., TORRES-CASTELLANOS, N., et al., "Tensile behavior of normal strength steel fiber green UHPFRC," doi:

http://doi.org/10.1016/j.conbuildmat.2021.122869. ACI Mater. J., v. 118, n. 1, pp. 127-138, 2021.

[18] ABELLÁN-GARCÍA, J., GUZMAN-GUZMAN, J.S., "Random forest-based optimization of UHPFRC under ductility requirements for seismic retrofitting applications,"

https://doi.org/10.1016/j.conbuildmat.2020.119465. Constr. Build. Mater., vol 285 (March 2020).

[19] TOLEDO FILHO, R.D., KOENDERS, E., FORMAGINI, S. et al., "Performance assessment of Ultra High Performance Fiber Reinforced Cementitious Composites in view of sustainability," doi: http://doi.org/10.1016/j.matdes.2011.09.022. Mater. Des., v. 36, pp. 880-888, 2012.

[20] ABELLÁN-GARCÍA, J., NÚÑEZ-LÓPEZ, A., TORRES-CASTELLANOS, N., et al., "Effect of FC3R on the properties of ultra-high-performance concrete with recycled glass", doi: http://doi.org/10.15446/dyna.v86n211.79596. v. 86, n. 211, pp. 84-92, 2019.

[21] ABELLÁN-GARCÍA, J., NÚÑEZ-LÓPEZ, A., TORRES-CASTELLANOS, N., et al., "Factorial design of reactive powder concrete containing electric arc slag furnace and recycled glass powder," doi: http://doi.org/10.15446/dyna.v87n213.82655. Dyna, vol. 87, no. 213, pp. 42-51, 202. 
[22] ABELLÁN, J., TORRES, N., NÚÑEZ, A., et al., "Concretos de muy altas prestaciones reforzados con fibras: estado del arte, aplicaciones y posibilidades en el mercado latinoamericano," in XXXVIII Jornadas Sudamericanas de ingeniería estructural, 2018.

[23] RAI. B., WILLE, K., "Develompment and testing of High / Ultra-High Early Stregnth Concrete," in Proceedings of Hipermat 2020 - 5th International Symposium on UHPC and Nanotechnology for Construction Materials, 2020, pp. 7-8.

[24] GHAFARI, E., COSTA, H., NUNO, E., et al. "Optimization of UHPC by Adding Nanomaterials," in 3rd International Symposium on UHPC and Nanotechnology for Construction Materials, Kassel Uni., Kassel, Germany, 2012, pp. 71-78.

[25] KALNY, M., KVASNICKA, V., KOMANEC, J., "First Practical Applications of UHPC in the Czech Republic," in Proceedings of Hipermat 2016 - 4th International Symposium on UHPC and Nanotechnology for Construction Materials, 2016, pp. 147-148.

[26] SHAABAN, M., AHMED, S., "Development of Ultra-High Performance Concrete Jointed Precast Decks and Concrete Piles in Integral Abutment Bridges," in The First International Symposium on Jointless \& Sustainable Bridges, 2016, n. May.

[27] HABER, Z.B., MUNOZ, J.F., GRAYBEAL, B.A., "Field Testing of an Ultra-High Performance Concrete Overlay (No. FHWA-HRT-17-096)", 2017.

[28] ACKER, P., BEHLOUL, M., "Ductal Technology: a Large Spectrum of Properties, a wide range of applications," in Proceedings of the International Symposium on Ultra High Performance Concrete, 2004, pp. $11-24$.

[29] ABELLÁN, J., NÚÑEZ, A., ARANGO, S., "Pedestrian bridge of UNAL in Manizales : A new UPHFRC application in the Colombian building market," in Proceedings of Hipermat 2020 - 5th International Symposium on UHPC and Nanotechnology for Construction Materials, 2020, n. July, pp. 43-44.

[30] TAYEH, B.A., ABU BAKAR, B.H., MEGAT JOHARI, M.A., et al., "Utilization of ultra-high performance fibre concrete (UHPFC) for rehabilitation a review," doi: http://doi.org/10.1016/j.proeng.2013.03.048. Procedia Eng., v. 54, pp. 525-538, 2013.

[31] ABELLÁN-GARCÍA, J., NUÑEZ-LOPEZ, A., ARANGO-CAMPO, S., Pedestrian Bridge over Las Vegas Avenue in Medellín. First Latin American Infrastructure in UHPFRC, in: P. Serna, A. Llano-Torre, J.R. Martí-Vargas, J. Navarro-Gregori (Eds.), BEFIB 2020, RILEM Bookseries, doi: https://doi.org/10.1007/978-3-030-58482-5_76. Valencia (Spain), 2020: pp. 864-872.

[32] DOGAN, E., KRSTULOVIC-OPARA, N., "Seismic Retrofit with Continuous Slurry-Infiltrated Mat Concrete Jackets," ACI Struct. J., v. 100, n. 6, 2003

[33] PARRA-MONTESINOS, G. J., "High-Performance Fiber-Reinforced Cement Composites : An Alternative for Seismic Design of Structures," ACI Struct. J., v. 102, n. 5, pp. 668-675, 2005.

[34] OLDEN, J.D., JACKSON, D.A., "Illuminating the 'black box': A randomization approach for understanding variable contributions in artificial neural networks," doi: http://doi.org/10.1016/S0304-

3800(02)00064-9. Ecol. Modell., v. 154, n. 1-2, pp. 135-150, 2002.

[35] OLDEN, J.D., JOY, M.K., DEATH, R.G., "An accurate comparison of methods for quantifying variable importance in artificial neural networks using simulated data," doi:

http://doi.org/10.1016/j.ecolmodel.2004.03.013. Ecol. Modell., v. 178, n. 3-4, pp. 389-397, 2004.

[36] FRANCESCHINI, S., GANDOLA, E., MARTINOLI, M., et al., "Cascaded neural networks improving fish species prediction accuracy: The role of the biotic information," doi: http://doi.org/10.1038/s41598-01822761-4. Sci. Rep., v. 8, n. 1, pp. 1-12, 2018.

[37] SCHMIDT, M., FEHLING, E., GEISENHANSLÜKEM, C., Eds., Proceedings of the International Symposium on Ultra High Performance Concrete. Kassel, Germany: University of Kassel, Germany, 2004.

[38] FEHLING, E., SCHMIDT, C., STÜWALD, S., Eds., Proceedings of the Second International Symposium on Ultra High Performance Concrete. University of Kassel, Germany, Kassel, Germany, 2008.

[39] SCHMIDT, M., FEHLING, E., GLOTZBACH, C., et al., , Eds., Proceedings of Hipermat 2012 3rd International Symposium on UHPC and Nanotechnology for High Performance Construction Materials, Kassel, Germany, 2012. 
[40] FEHLING, E., MIDDENDORF, B., J. THIEMICKE, J., Eds., Proceedings of Hipermat 2016 4th International Symposium on UHPC and Nanotechnology for High Performance Construction Materials. Kassel, Germany: University of Kassel, Germany, 2016.

[41] DE LARRARD, F., "Concrete mixture proportioning: a scientific approach., in Modern concrete technology series,” E\&FN SPON., 1999.

[42] DE LARRARD, F., "Optimization of Ultra-High Performance Concrete by the Use of a Packing Model," Cem. Concr. Res., v. 24, n. 6, pp. 997-1009, 1994.

[43] KORPA, A., KOWALD, T., TRETTIN, R., "Principles of development, phase composition and nanostructural features of multiscale Ultra High Performance Concrete modified with pyrogenic nanoparticles - A review article,” Am. J. Mater. Sci. Appl., v. 2, n. 2, pp. 17-30, 2014.

[44] CAMACHO TORREGROSA, E., "Dosage optimization and bolted connections for UHPFRC ties," Polytechnic University of Valencia, Spain, 2013.

[45] SKAZLIC, M., BJEGOVIC, D., SERDAR,M., "Influence of test specimens geometry on compressive strength of ultra-high performance concrete," in Proceedings of the 2nd International Symposium on Ultra High Performance Concrete, Kassel, Germany, 2008, pp. 295-301.

[46] NAAMAN, A.E., WILLE, K., "Some correlation between hihg packing density, ultra-high performance, flow hability, and fiber reinforcement of a concrete matrix, in bac2010," Congresso Ibêrico sobre betão autocompactável, 2010.

[47] GRAYBEAL, B., DAVIS, M., "Cylinder or Cube : Strength Testing of 80 to 200 Fiber-Reinforced Concrete Cylinder or Cube : Strength Testing of 80 to $200 \mathrm{MPa}$ ( 11 . 6 to $29 \mathrm{ksi}$ ) Ultra-High-Performance FiberReinforced Concrete," ACI Mater. J., v. 105, n. 6, pp. 603-609, 2014.

[48] ATKINSON, A., RIANI, M., "Robust Diagnostic Regression Analysis." New York: Springer US, 2000.

[49] HÄRDLE, W.K., SIMAR, L., “Applied Multivariate Statistical Analysis.” Berlin: Springer-Verlag $\mathrm{GmbH}, 2012$.

[50] EVERITT, B., HOTHORN, T., "MVA: An Introduction to Applied Multivariate Analysis with R," 2015.

[51] ARIZZI, A., CULTRONE, G., "Comparing the pozzolanic activity of aerial lime mortars made with metakaolin and fluid catalytic cracking catalyst residue : A petrographic and physical-mechanical study," doi: http://doi.org/10.1016/j.conbuildmat.2018.07.002. Constr. Build. Mater., v. 184, pp. 382-390, 2018.

[52] TORRES CASTELLANOS, N., "Estudio en estado fresco y endurecido de concretos adicionados con catalizados de craqueo catalítico usado (FCC)," Universidad Nacional de Colombia, 2014.

[53] LI, Z., RANGARAJU, P.R., "Development of UHPC Using Ternary Blends of Ultra- Fine Class F Fly Ash , Metakaolin and Portland Cement," in First International Interactive Symposium on UHPC, 2016, n. September, pp. 1-12.

[54] FERDOSIAN, I., CAMÕES, A., RIBEIRO, M., "High-volume fly ash paste for developing ultra-high performance concrete (UHPC)," doi: http://doi.org/10.1016/j.ctmat.2016.10.001. Cienc. e Tecnol. dos Mater., v. 29, n. 1 , pp. $157-161,2017$.

[55] CHANDWANI, V. AGRAWAL, V. NAGAR, R., "Modeling slump of ready mix concrete using genetic algorithms assisted training of Artificial Neural Networks," doi: http://doi.org/10.1016/j.eswa.2014.08.048. Expert Syst. Appl., v. 42, n. 2, pp. 885-893, 2015.

[56] CHOLLET, F., ALLAIRE, J.J., “Deep Learning with R.” New Jersey: Manning Publications Co., 2018.

[57] ABELLÁN-GARCÍA, J., FERNÁNDEZ-GÓMEZ, J.A., TORRES-CASTELLANOS, et al. "Machine Learning Prediction of Flexural Behavior of UHPFRC," in: P. Serna, A. Llano-Torre, J.R. Martí-Vargas, J. Navarro-Gregori (Eds.), Fibre Reinf. Concr. Improv. Innov. BEFIB 2020., RILEM Bookseries, doi: http://doi.org/10.1007/978-3-030-58482-5_52. Valencia, Spain, 2020: pp. 570-583.

[58] ABELLÁN-GARCÍA, J., "Dosage optimization and seismic retrofitting applications of Ultra-HighPerformance Fiber Reinforced Concrete (UHPFRC)," Universidad Politécnica de Madrid, 2020.

[59] ABELLÁN-GARCÍA, J., "Comparison of artificial intelligence and multivariate regression in modeling the flexural behavior of UHPFRC", doi: http://doi.org/10.15446/dyna.v87n214.86172. v. 87, n. 214, pp. 239$248,2020$. 
[60] ADELI, H., "Neural Networks in Civil Engineering : 1989 - 2000," Comput. Civ. Infrastruct. Eng., v. 16, pp. 126-142, 2001.

[61] ROSENBLATT, F., "The Perceptron: A probabilistic model for information storage and organization in the brain," Cornerr Aeronaut. Lab., v. 65, n. 6, pp. 386-408, 1958.

[62] ANDERSON, J.A., "Cognitive and Psychological Computation with Neural Models,” IEEE Trans. Syst. Man. Cybern., v. 13, n. 5, pp. 799-816, 1983.

[63] BHARATHI, S.D., MANJU, R., PREMALATHA, J., "Prediction of Compressive Strength for SelfCompacting Concrete (SCC) using Artificial Intelligence and Regression Analysis," Int. J. ChemTech Res., v. 10, n. 8, pp. 263-275, 2017.

[64] DEMIR, F., "Prediction of elastic modulus of normal and high strength concrete by artificial neural networks," Construction and Building Materials, v. 22, pp. 1428-1435, 2008.

[65] ESTEBON, M..D. "Perceptrons : An Associative Learning Network” by, Virginia Tech, no. June 1960, 1997.

[66] MUSHGIL, H.M., ALANI, H.A., GEORGE, L.E., "Comparison between Resilient and Standard Back Propagation Algorithms Efficiency in Pattern Recognition,” Int. J. Sci. Eng. Res., v. 6, n. 3, pp. 773-778, 2015.

[67] PRASAD, N., SINGH, R., LAL, S.P., "Comparison of back propagation and resilient propagation algorithm for spam classification," doi: http://doi.org/10.1109/CIMSim.2013.14. Proc. Int. Conf. Comput. Intell. Model. Simul., pp. 29-34, 2013.

[68] DE LARRARD, F., SEDRAN, T., "Optimization of ultra-high-performance concrete by the use of a packing model," doi: http://doi.org/10.1016/0008-8846(94)90022-1. Cem. Concr. Res., v. 24, n. 6, pp. 9971009, 1994.

[69] R CORE TEAM, "R: A Language and Environment for Statistical Computing.” Vienna, Austria, 2018, https://www.r-project.org/

[70] GÜNTHER, F., FRITSCH, S., “neuralnet: Training of Neural Networks,” $R$ J., v. 2, n. 1, pp. 30-38, 2010, doi: http://doi.org/10.1109/SP.2010.25

[71] MORIASI, D.N., ARNOLD, J.G., VAN LIEW, M.W., et al., "Model evaluation guidelines for systematic quantification of accuracy in watershed simulations," Am. Soc. Agric. Biol. Eng., v. 50, n. 3, pp. 885-900, 2007.

[72] NASH, E., SUTCLIFFE, V., "River flow forecasting through conceptual models: Part I - A discussion of principles," J. Hydrol., v. 10, pp. 282-290, 1970.

[73] SRINIVASULU, S., JAIN, A., "A comparative analysis of training methods for artificial neural network rainfall - runoff models," doi: http://doi.org/10.1016/j.asoc.2005.02.002. Appl. Soft Comput., v. 6, pp. 295306, 2006.

[74] GUPTA, S., "Using Artificial Neural Network to Predict the Compressive Strength of Concrete containing Nano-silica,” doi: http://doi.org/10.13189/cea.2013.010306. Civ. Eng. Archit., v. 1, n. 3, pp. 96-102, 2013.

[75] ABELLÁN, J., FERNÁNDEZ, J., TORRES, N., "Properties prediction of environmentally friendly ultra-high-performance concrete using artificial neural networks,"

https://doi.org/10.1080/19648189.2020.1762749. European Journal of Environmental and Civil Engineering, v. 24, n. 6, May 2020.

[76] ABELLÁN-GARCÍA J., "Four-layer perceptron approach for strength prediction of UHPC" https://doi.org/10.1016/j.conbuildmat.2020.119465. Constr. Build. Mater., v. 256, September 2020.

[77] SHI, C., WU, Z., XIAO, J., et al., “A review on ultra high performance concrete: Part II. Hydration, microstructure and properties," doi: http://doi.org/10.1016/j.conbuildmat.2015.10.088. Constr. Build. Mater., v. 101, n. October, pp. 741-751, 2015.

[78] ZHANG, J., ZHAO, Y., "Experimental Investigation and Prediction of Compressive Strength of UltraHigh Performance Concrete (UHPC) Containing Supplementary Cementitious Materials," doi: http://doi.org/10.1155/2017/4563164. Hindawi Adv. Mater. Sci. Eng., v. 2017, pp. 522-525, 2017. 
[79] GHAFARI, E., BANDARABADI, M., COSTA, H., et al., "Prediction of Fresh and Hardened State Properties of UHPC: Comparative Study of Statistical Mixture Design and an Artificial Neural Network Model," doi: http://doi.org/10.1061/(ASCE)MT.1943-5533.0001270. J. Mater. Civ. Eng., v. 27, n. 11, p. $04015017,2015$.

[80] KHATIB, J.M., WILD, S., "Pore size distribution of metakaolin paste," doi: http://doi.org/10.1017/CBO9781107415324.004. Cem. Concr. Compos., v. 26, n. 10, pp. 1689-1699, 1996.

[81] POON, C.S., LAM, L., KOU, S.C., et al., "Rate of pozzolanic reaction of metakaolin in highperformance cement pastes," doi: http://doi.org/10.1016/S0008-8846(01)00581-6. Cem. Concr. Res., v. 31, n. 9, pp. 1301-1306, 2001.

[82] ABELLAN-GARCIA, J., SANTOFIMO-VARGAS, M.A., TORRES-CASTELLANOS, N., “Analysis of metakaolin as partial substitution of ordinary Portland cement in Reactive Powder Concrete," Adv. Civ. Eng. Mater, in press.

[83] BEHNOOD, A., ZIARI, H., "Effects of silica fume addition and water to cement ratio on the properties of high-strength concrete after exposure to high temperatures," doi: http://doi.org/10.1016/j.cemconcomp.2007.06.003. Cem. Concr. Compos., v. 30, n. 2, pp. 106-112, 2008.

[84] VAN TUAN, N., YE, G., VAN BREUGEL, K., et al., "The study of using rice husk ash to produce ultra high performance concrete, doi: http://doi.org/10.1016/j.conbuildmat.2010.11.046. Constr. Build. Mater., v. 25, n. 4, pp. 2030-2035, 2011.

[85] CORREA-YEPES, J.A., ROJAS-REYES, N.R., TOBÓN, J.I., "Effect of fly ash and silica fume on rheology, compressive strength and self-compacting in cement mixtures," doi: http://doi.org/10.15446/dyna.v85n206.68960. Dyna, v. 85, n. 206, pp. 59-68, 2018.

[86] MENG, W., SAMARANAYAKE, V.A., KHAYAT, K.H., "Factorial design and optimization of UHPC with lightweight sand," doi: http://doi.org/10.14359/51700995. ACI Mater. J., n. February, 2018.

[87] AHMAD, S., HAKEEM, I., MASLEHUDDIN, M., "Development of UHPC Mixtures Utilizing Natural and Industrial Waste Materials as Partial Replacements of Silica Fume and Sand," doi: http://doi.org/10.1155/2014/713531. European Journal of Environmental and Civil Engineering, v. 2014, 2014.

[88] TURK, K., DEMIRHAN, S., "Effect of limestone powder on the rheological, mechanical and durability properties of ECC," doi: http://doi.org/10.1080/19648189.2016.1150902. Eur. J. Environ. Civ. Eng., v. 21, n. 9, pp. 1151-1170, 2017.

[89] GHAFOORI, N., SPITEK, R., NAJIMI, M., "Influence of limestone size and content on transport properties of self-consolidating concrete," doi: http://doi.org/10.1016/j.conbuildmat.2016.10.051. Constr. Build. Mater., v. 127, pp. 588-595, 2016.

[90] MOSABERPANAH, M.A., EREN, O., "Effect of quartz powder, quartz sand and water curing regimes on mechanical properties of UHPC using response surface modeling," doi: http://doi.org/10.12989/acc.2017.5.5.481. Adv. Concr. Constr., v. 5, n. 5, pp. 481-492, 2017.

[91] RUSSEL, H., GRAYBEAL, B, "Ultra-High Performance Concrete : A State-of-the-Art Report for the Bridge Community," 2013.

[92] PUERTAS, F., SANTOS, H., PALACIOS, et al., "Polycarboxylate superplasticiser admixtures: effect on hydration, microstructure and rheological behaviour in cement pastes," doi: http://doi.org/10.1680/adcr.17.2.77.65044. Adv. Cem. Res., v. 17, n. 2, pp. 77-89, 2005.

[93] KUBENS, S., "Interaction of cement and admixtures and its influence on rheological properties," Göttingen, Germany, 2010.

[94] GHAFARI, E., COSTA, H., JÚLIO, E., et al., "Enhanced Durability of Ultra High Performance Concrete by Incorporating Supplementary Cementitious Materials," Second Int. Conf. Microestructural-related Durab. Cem. Compos., no. November 2014, pp. 11-13, 2012.

[95] ABELLÁN-GARCÍA, J., TORRES-CASTELLANOS, N., FERNÁNDEZ-GÓMEZ, J.A., et al., "Ultrahigh-performance concrete with local high unburned carbon fly ash," doi: http://doi.org/10.15446/dyna.v88n216.89234. Dyna., v. 88, pp 38-47,2021. 
[96] MAHMUD, H.B., BAHRI, S., YEE, Y.W., et al., "Effect of Rice Husk Ash on Strength and Durability of High Strength High Performance Concrete," World Acad. Sci. Eng. Technol., v. 2016, n. 3, pp. 390-395, 2016.

\section{ORCID}

Joaquin Abellan García

https://orcid.org/0000-0002-0353-322X 